\title{
An Introduction to Fuzzy Topological Spaces
}

\author{
I. Zahan, R. Nasrin* \\ Department of Mathematics, Bangladesh University of Engineering \& Technology, Dhaka, Bangladesh \\ Email: ^rehena@math.buet.ac.bd
}

How to cite this paper: Zahan, I. and Nasrin, R. (2021) An Introduction to Fuzzy Topological Spaces. Advances in Pure Mathematics, 11, 483-501.

https://doi.org/10.4236/apm.2021.115034

Received: December 9, 2020

Accepted: May 22, 2021

Published: May 25, 2021

Copyright (c) 2021 by author(s) and Scientific Research Publishing Inc. This work is licensed under the Creative Commons Attribution International License (CC BY 4.0).

http://creativecommons.org/licenses/by/4.0/

\begin{abstract}
Topology has enormous applications on fuzzy set. An attention can be brought to the mathematicians about these topological applications on fuzzy set by this article. In this research, first we have classified the fuzzy sets and topological spaces, and then we have made relation between elements of them. For expediency, with mathematical view few basic definitions about crisp set and fuzzy set have been recalled. Then we have discussed about topological spaces. Finally, in the last section, the fuzzy topological spaces which is our main object we have developed the relation between fuzzy sets and topological spaces. Moreover, this article has been concluded with the examination of some of its properties and certain relationships among the closure of these spaces.
\end{abstract}

\section{Keywords}

Topological Space, Fuzzy Set, Topological Fuzzy Set, Closure Fuzzy

Topological Set

\section{Introduction}

Primarily in the area of set theory, fuzzy mathematics differs from conventional mathematics. Fuzzy mathematics had been introduced just few years ago, it is full of topics. It is used widely in many sectors such as, vehicles, traffic system where logic circuit controls anti-skid brakes, transmissions, and other operations. We have discussed in this paper about a set, which is more specified than crisp set. It can take a decision between yes or no, i.e. 1 or 0 . Thirty years ago, Black [1], a philosopher in America, predicted some ideas which are obtainable in this article. The author of [1] established a hypothesis whose main things fuzzy sets-the sets having borders which are not specific. An influential paper by Zadeh [2] provided a vital point regarding the development of the up to date ideas of ambiguity. 
In this real physical world, it would be better if we are able to study the objects in a classified way. But most often than not, it cannot be done, because the objects do not exist a specifically definite criterion of association. Many illustrations may be written, the group of animals obviously consists of birds, cats, deer, etc. On the other hand, the things like bacteria, virus, starfish, jellyfish, etc., have an uncertain category regarding the animal's class. The similar type of uncertainty arises for a numerical value like 10 regarding to the "class" of the set of all real values which become higher than 1 . Obviously, "the group of the set of all real values which become higher than 1" otherwise "the class of beautiful women" otherwise "the class of tall men," can not represent sets otherwise groups in the common mathematical logic of these languages. However, the truth continues that such inaccurately called "classes" take part in a vital position in human being thoughts, mainly for the area of prototype abstraction, informative communication and recognition. Research based on the fuzzy sets hypothesis is increasing gradually from the time at the beginning of the hypothesis in mid 1960s. Now, the concepts and outcomes containing the hypothesis of fuzzy set are relatively remarkable. In addition, various applications based research has been conducted very vigorously and has found more extraordinary outcomes.

For conciseness, the concepts of the definition of topology on fuzzy sets that may be named as topological fuzzy spaces, Chang [3] confined his attention to the more basic definitions, theorems and proofs. Michálek [4] identified and analyzed another idea of topological fuzzy space in his paper which was quite different of the classic Chang's [3] definition. Lowen [5] provided profound concepts for the construction of topological fuzzy spaces. He introduced two new functions and served the idea of fuzzy compression for the simplification of topological compression which allowed seeing obviously additional relation amid topological fuzzy spaces and topological spaces. Hutton [6] continued his study to find the real meaning of the common theorems of topology, and then took a broad view of their proofs. He also developed "pointless" descriptions for structures and properties that depend merely on the Lattice composition of fuzzy sets compilation, but not on its putrefaction. Cheng-Ming [7] discussed about the product-induced spaces, which is a special fuzzy topological spaces. $\mathrm{He}$ showed that each topological fuzzy space is isomorphic topologically by a definite space of topology and also introduced the basic idea of double points and set up a type of fuzzy points neighborhood formation for example the Q-neighborhood, which is very significant conception in topological fuzzy set. Finally he discussed the dilemma of metrization in fuzzy on topological fuzzy spaces in addition to obtain a metrization hypothesis in fuzzy. Zimmermann [8] in his book presented the detail of fuzzy set theory and their applications. Papageorgiou [9] introduced several fuzzy topological concepts, fuzzy multifunction and the conception of neighborhood point in fuzzy which is helpful in the learning of common optimization techniques and games in fuzzy. He remarked in his study that common topological point set may be considered as a particular form of topological fuzzy, 
wherever the entire functions of relationship are only functions of characteristic.

Klir and Yuan [10] provided the details of the theoretical advances in fuzzy logic and set theory in his book. The author [10] considered an extensive diversity of usages of both fuzzy logic and sets. Many topological properties for intuitionistic I-topological spaces have been discussed in Lee and Lee [11] and Yang and Wang [12]. The authors [12] proved that the accompaniment of any closed set is an open set and the opposite of this statement is also true. They also demonstrated an illustration for computing the exterior, interior, and boundary of Mikania micrantha pedestaled on aerial snaps of Hong Kong landscape. Wenzhong and Kimfung [13] developed the concept of arithmetical topological fuzzy pedestaled on the closure and interior operators. Next, coherent topological fuzzy is named by these operators. Separation and regularity axioms in fuzzy topology on fuzzy set and their characterizations are defined and studied by Kandil et al. [14]. Moreover, with some necessary examples they investigated some of their basic properties and certain relationship among them. Using a special kind of function Akray [15] introduced three different fuzzy topological spaces. In this study he discussed compactness, connectedness properties and also provides the necessary and sufficient conditions under which properties some of these spaces coincide. Aygunoglu et al. [16] investigated the usages of theory of Soft Set on actual life dilemmas and different disciplines. The authors [16] investigated some of its fundamental properties and concluded that soft fuzzy topology and soft fuzzy theory may be well-matched. Nowadays more advanced studies are happing on the application of fuzzy topological spaces. Chamuah and Chetia [17] provided a proper description of the arithmetical topological fuzzy pedestaled on the closure and interior operators. They presented in their study an illustration for computing exterior and interior boundaries of India's flood influenced regions

To complete the study, first we discussed the concepts of fuzzy sets in Section 2, then we discussed topological space in Section 3, and in Section 4 we mainly classify fuzzy topological spaces, which is our main object. For this we have discussed some important definitions and related theorems. To complete the Section 2 we have used the following references [8] [9], and to complete the Section 3 we have used the following references [18] [19] [20].

Finally, we deal with the topics of topological fuzzy spaces. In this research, the definition and concept of topological fuzzy spaces with examples, closure topological fuzzy spaces, consequent properties of closure operator with proof and theorem, interior of topological fuzzy spaces, fuzzy points, neighborhood of topological fuzzy spaces with example, and corresponding theorem, inverse function of a topological fuzzy spaces, continuous function of a topological fuzzy spaces, and corresponding theorem have been discussed. We also discussed about dense, base, subbase of topological fuzzy spaces with example and lastly Hausdroff fuzzy topological spaces and their theorem. We also have showed some related theorem of these topics. 


\section{Fuzzy Sets}

Fuzzy set is the more universal concept of classical set which is an impending tool for handling indistinctness and uncertainties. It is typically characterized in the form of membership function, whereas a membership function is characterized by the universal set $\tilde{U}$ to the set ranging between 0 \& 1 .

More exclusively, let $I=[0,1]$ be the unit interval and $X$ be a null set, where $x$ be any particular element in $X$. Subsequently, a function $\mu: X \rightarrow I$, where $x \rightarrow \mu(x)$ is described as fuzzy set in $X$. Where, $\mu(x)$ is defined as the "Grade of membership of $x \in X$ in $\mu$ "

\section{Example 1:}

In the example three consequences fuzzy sets are explain that stand for the perception of very young, young and middle-age person in a country which also mentioned graphically in Figure 1. The membership functions $\alpha, \beta$ and $\chi$ are stand for these concepts defined on the interval $[0,50]$ as follows:

$$
\begin{aligned}
& \alpha(x)=\left\{\begin{array}{l}
1, \text { when } x \leq 15 \\
(25-x) / 10, \text { when } 15<x<25 \\
0, \text { otherwise }
\end{array}\right. \\
& \beta(x)=\left\{\begin{array}{l}
0, \text { when } x \leq 15 \text { or } x>40 \\
(x-15) / 10, \text { when } 15<x<25 \\
1, \text { if } 25 \leq x \leq 40
\end{array}\right. \text { and } \\
& \chi(x)=\left\{\begin{array}{l}
0, \text { when } x \leq 35 \\
(x-35) / 10, \text { when } 35<x<45 \\
1, \text { otherwise }
\end{array}\right.
\end{aligned}
$$

\subsection{Some Definitions}

\subsubsection{Crisp Set}

Crisp set is a group of objects that define the precious and definite feature which employs bi-valued (yes/no) logic. That is whether each particular element can either within or not belong to a set $S, S \subseteq X$ It is mainly a classical set which is label by a special type of fuzzy sets. Crisp set can be denoted by

$$
\chi_{s}: X \rightarrow\{0,1\}
$$

\section{Example:}

The set of teenagers is an example of crisp set.

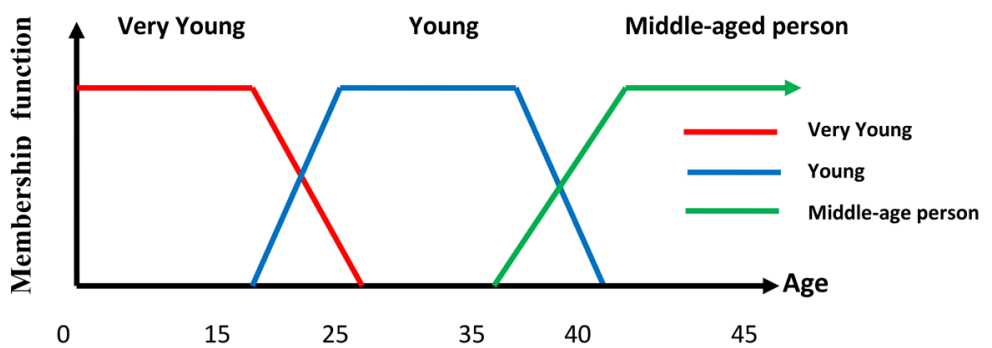

Figure 1. Fuzzy sets. 


\subsubsection{Classical Set}

A collection of individual object identified as the member or elements of the set that can be distinguished from one another and which follows some basic property is known as classical set. It is defined in such a way that, each element of the set is spitted either member or non-member groups. i.e. for a set $A$ either $a \in A$ or $a \notin A$. There is no chance of existence of partial membership.

\section{Example:}

For instance, $2 \in\{1,2,3,4,5,7,9\}$, and $5 \notin\{1,2,3\}$.

\subsubsection{Characteristic Function of Crisp Sets}

Suppose the set of universal set is denoted by $X$ and $S$ is the subset of $X$, i.e. $\phi$. Then for each $x \in X$ its characteristic function is denoted by $\lambda_{S}$ or $1_{S}$ is defined as,

$$
1_{S}(x)=\lambda_{S}(x)= \begin{cases}0 ; & \text { if } x \notin S \\ 1 ; & \text { if } x \in S\end{cases}
$$

i.e. when $\lambda_{S}(x)=1 \Rightarrow x \in S$, and $\lambda_{S}(x)=0 \Rightarrow x \notin S$.

Figure 2 show the graphical representation of the above example.

\subsubsection{Characteristic Function of the Complement}

For a set $S$ the complement of the characteristic function is denoted by $1_{\bar{S}}$ or $\lambda_{\bar{S}}$ and for each $x \in X$ defined as

$$
1_{\bar{S}}(x)=1-1_{\bar{S}}(x)=\left\{\begin{array}{l}
0, \text { if } x \in S \\
1, \text { if } x \notin S
\end{array}\right.
$$

\section{Example:}

$$
\text { Let } 1_{\bar{S}}(x)=\left\{\begin{array}{l}
1, \text { if } 3 \leq x \leq 10 \\
0, \text { otherwise }
\end{array}\right.
$$

Therefore,

$$
1_{\bar{S}}(x)=\left\{\begin{array}{l}
0, \text { if } 3 \leq x \leq 10 \\
1, \text { if } 0 \leq x<3 \text { or } 10<x<\infty
\end{array}\right.
$$

Figure 3 express the graphical representation of the above example

\subsubsection{Characteristic Functions (Union and Intersection)}

Let $P$ and $X=\{1,2,3,4,5\}$ be two sets, the characteristic functions of the union and intersection are denoted by $1_{P \cup Q}$ and $1_{P \cap Q}$ can also be obtain by pertaining the formulas:

$$
1_{P \cup Q}(x)=\max \left[1_{P}(x), 1_{Q}(x)\right] \& 1_{P \cap Q}(x)=\min \left[1_{P}(x), 1_{Q}(x)\right] \text {, respectively. }
$$

\section{Example:}

Let, $P=\{x: 5 \leq x \leq 15\}, Q=\{x: 10 \leq x \leq 20\}$

$$
\therefore 1_{P}(x)=\left\{\begin{array}{l}
1, \text { if } 5 \leq x \leq 15 \\
0, \text { otherwise }
\end{array} \& 1_{Q}(x)=\left\{\begin{array}{l}
1, \text { if } 10 \leq x \leq 20 \\
0, \text { otherwise }
\end{array}\right.\right.
$$

So, $1_{P \cup Q}(x)=\left\{\begin{array}{l}1, \text { when } 5 \leq x \leq 10 \\ 0, \text { otherwise }\end{array} \& 1_{P \cap Q}(x)=\left\{\begin{array}{l}1, \text { when } 10 \leq x \leq 15 \\ 0 \text {, otherwise }\end{array}\right.\right.$

Figure 4 displays the graphical representation of the above example 


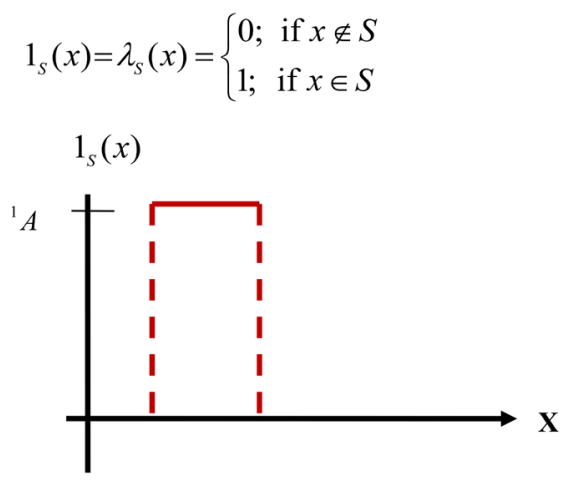

Figure 2. Where "0" and "1" denote excluded and included values respectively.
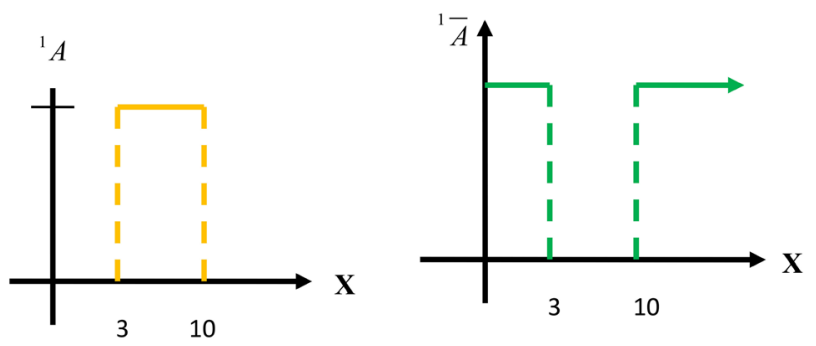

Figure 3. Characteristic function of the complement.
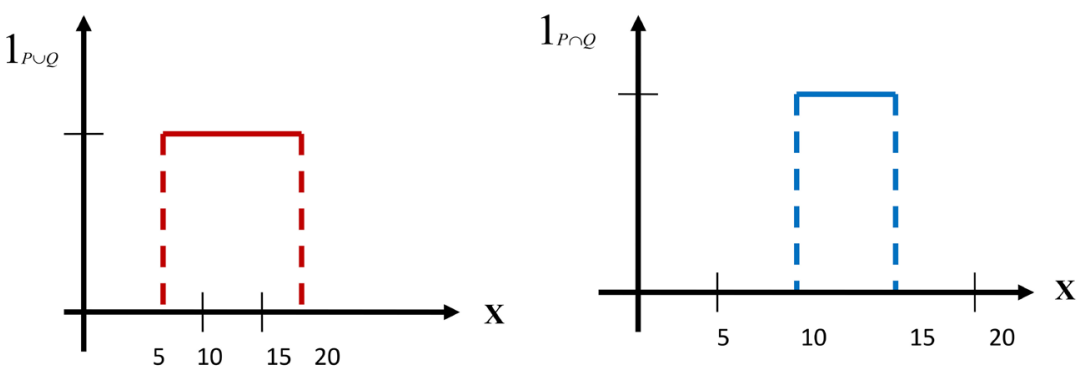

Figure 4. Characteristic function of $1_{A \cap B}$ and $1_{A \cup B}$ respectively.

\subsection{Standard Fuzzy Operations}

The most three operations that has a special significance in fuzzy set theory are $t$.

1) union 2) intersection and, 3) complement.

These can be generalized to fuzzy sets in many ways.

\subsubsection{Standard Fuzzy Union and Intersection}

Let $A$ and $B$ be two fuzzy set which is defined on $X$, where $X$ defined the universal set.

Then, the standard fuzzy union of $A$ and $B$, denoted by $A \vee B$ and can be described as

$$
\mu_{A \cup B}=\max \left[\mu_{A}(x), \mu_{B}(x)\right], \text { for all } x \in X .
$$

where, "max" indicates for maximum value.

and for the set $A$ and $B$ the standard fuzzy intersection is denoted by $A \wedge B$ 
is defined by via the formula,

$$
\mu_{A \cap B}=\min \left[\mu_{A}(x), \mu_{B}(x)\right] \text {, for all } x \in X .
$$

where, "min" stands for minimum value.

\subsubsection{Standard Fuzzy Complement}

If $A$ is any fuzzy set which is defined on a universal set $X$, then its complement denoted by $\bar{A}$ is another fuzzy set on $X$. So, the membership function of the complement of the fuzzy set $A$ is symbolized by $\mu_{A}: X \rightarrow[0,1]$ and described as

$$
\mu_{\bar{A}}=1-\mu_{A}(x), \quad \forall x \in X
$$

\section{Example:}

To demonstrate the significance of this definition, let us consider the fuzzy set $B$ of experienced postgraduate students, whose membership function is given in Figure 5.

\section{Topological Space}

Topology which is a basic mathematical discipline and whose name was not coined until 1930s has now its influence on many branches of pure mathematics especially geometry and analysis and some of the applied too. We have just used the word topology in its primary sense, as the name of a branch of mathematics. This word derives from two Greek words, and its literal meaning is "the science of position". In recent times for both graduate and undergraduate students, general topology has become a crucial part in Mathematics. For some time now, topology has been firmly established as one of the basic disciplines of pure mathematics. It has also deeply stimulated the abstract algebra and fuzzy mathematics.

\subsection{Basic Ideas and Definitions}

Suppose $X$ be a non empty set, a class $\tau$ which is a subset of $X$ is defined a topology if it satisfies the following conditions:

(i) $X$ and $\varphi$ belong to $\tau$.

(ii) The union and intersection of any number of sets in $\tau$ belongs to $\tau$.

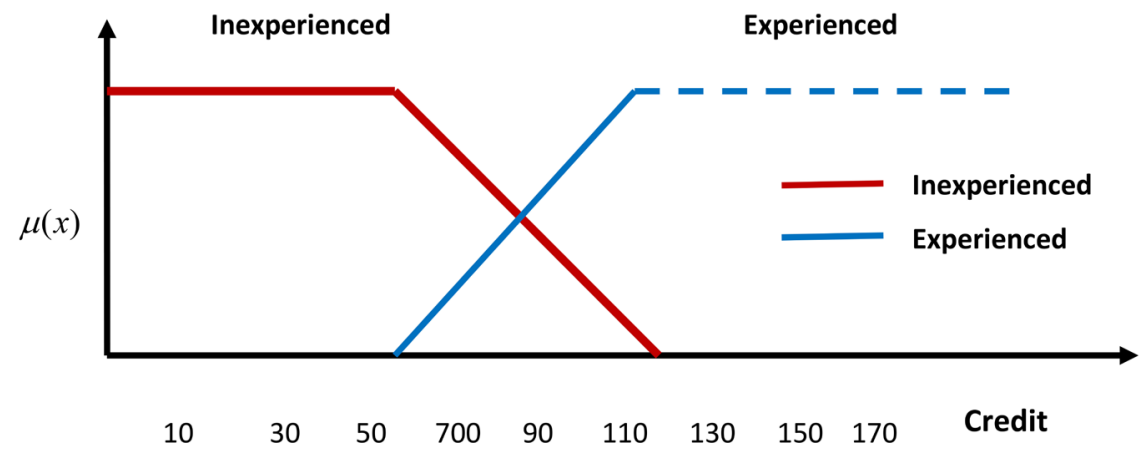

Figure 5. The set of experienced undergraduate students and its complement. 
The pair $(X, \tau)$ is called a topological space and the number of $\tau$ are described as $\tau$-open sets in $X$.

Example:

For any subsets of $X=\{1,2,3,4,5\}$.

$$
\begin{aligned}
& \tau_{1}=\{X, \varphi,\{1\},\{3,4\},\{1,3,4\},\{2,3,4,5\}\} \\
& \tau_{2}=\{X, \varphi,\{1\},\{3,4\},\{1,3,4\},\{2,3,4\}\} \\
& \tau_{3}=\{X, \varphi,\{1\},\{3,4\},\{1,3,4\},\{1,2,4,5\}\}
\end{aligned}
$$

We noticed that $\tau_{1}$ is a topology on $X$ but $\tau_{2}$ and $\tau_{3}$ is not a topology on $X$. Since $\tau_{1}$ satisfies the necessary two conditions (i), and (ii) where as the union $\{1,3,4\}\{2,3,4\}=\{1,2,3,4\}$ does not belong to $\tau_{2}$ and the intersection $\{1,3,4\}\{1,2,4,5\}=\{1,4\}$ of two sets in $\tau_{3}$ does not belong to $\tau_{3}$ i.e. $\tau_{2}$ and $\tau_{3}$ does not satisfy the condition (ii).

\section{Discrete topology}

Assume $X$ be a nonempty set. Then the group of all subsets including the empty set, of $X$, known as Power set $P(X)$ is a topology on $X$ and is define as discrete topology.

Example:

If $X=\{p, q\}$, and $\tau=\{X,\{p\},\{q\}\}$.

Then $\tau$ is discrete topology on $X$.

\section{Indiscrete topology}

For any non empty set $X$ the collection of set consisting $\phi$ and $X$ is a topology on $X$, is defined as indiscrete topology.

\section{Example:}

Let, $X=\{p, q, r\}$ then $\tau=\{X, \phi\}$ is a topology on $X$ and is known as indiscrete topology.

\subsection{Interior, Exterior and Boundary Points}

For a open set $x \in E \subseteq B$ a point $x \in B$ is an interior point iff $x \in E \subseteq B$ which is denoted by $x \in E \subseteq B \operatorname{int}(B)$ where $B$ is a subset of topological space. The interior of $B^{C}$ is known as the exterior of $B$ and is denoted by ext. The point which is interior nor an exterior of $B$ is known as boundary point of $B$.

i.e. $\operatorname{bd}(B)=$ Complement of $(\operatorname{int}(B) \cup \operatorname{ext}(B))$.

\subsection{Closure of a Set}

The intersection of all closed super sets of $B$ is denoted by $\bar{B}$ or $B^{-}$is known as closure of $B$.

Where, $(X, \tau)$ is the topological space and $B$ is the subset of it. If $\left\{E_{i}: i \in I\right\}$, denote the class of all closed subsets of $X$ containing $B$, then $\bar{B}$ will be the closure of a set $B$, if

(i) $\bar{B}$ is closed and $B=\bar{B}$.

(ii) if $E$ is a closed super set of $B$, then $B \subset \bar{B} \subset E$.

\section{Properties of closure operator}


For any topological space $(X, \tau)$ if $P$ and $Q$ be two arbitrary subsets of $X$, then the operation of forming closures hold the following four properties:

(i) $\bar{\varphi}=\varphi$;

(ii) $P \subseteq \bar{P}$;

(iii) $\overline{\bar{P}}=\bar{P}$;

(iv) $\overline{P \cup Q}=\bar{P} \cup \bar{Q}$.

\subsection{Homomorphism Topological Spaces}

A continuous one-to-one mapping of a topological space onto another is defined to be homomorphic if there exist a homeomorphism of $X$ onto $Y$ and their points can be set into one-to-one correspondence in such a manner that their open sets also correspond to one another.

\section{Fuzzy Topological Spaces}

The perception of Fuzzy set theory bring in [2] provides us a wider structure compared to classical set theory which generalize various concepts of topology. Fuzzy topology merges ordered structure to the topological structure. From the point of pure mathematics, this branch of mathematics was first proposed by enormous Mathematician Ehrenman who encompasses the two most active features of topology on lattice, which affect each other. Chang [3] had introduced first the notation of Fuzzy topology. Later on, numerous of researchers continued the study in this area. We observed that fuzzy topology is consider as special case of general point set topology, where membership functions are presently by characteristic functions.

\subsection{Basic Definitions}

In the past, the leading attempt to build up the fuzzy counterpart of basic topology was commenced by Chang [7] in 1968. From the point of Chang, on a set $X$ a fuzzy topology is a family $F=\{\mu: \mu$ is fuzzy set in $X\}$ of fuzzy subsets (i.e. $\left.F \in I^{X}\right)$ that satisfies the following three axioms:

(i) $0,1 \in F$;

(ii) $\mu_{1}, \mu_{2} \in F$, then $\mu_{1} \wedge \mu_{2} \in F$;

(iii) If $\left\{\mu_{i}: i \in j\right\} \subset F$, where $j$ denotes an index set, then $\vee \mu_{i} \in F$;

$F$ is described as a fuzzy topology for $X$ and the pair $(X, F)$ is named as a fuzzy topological space or in short f.t.s. The members of $F$ are defined as $F$-open fuzzy set. If the complement of $\rho$, denoted by $\rho^{C}$, is $F$-open then an element $\rho \in[0,1]^{X}$ is said to be a closed fuzzy set.

\section{Indiscrete Fuzzy topology}

The same as in general fuzzy topology, the indiscrete fuzzy topology contains only fuzzy sets 0 and 1 .

\section{Example 1:}

Indiscrete fuzzy topological $(X, F), F=\left\{\rho \in I^{X}: \alpha\right.$ constant fuzzy set $\}$ i.e. for all, $\rho \in F$, if $\rho=$ constant 
Then, $\rho(x)=0, \forall x$

$$
\rho(x)=1, \forall x
$$

Wherever, $I^{X}$ is the fuzzy set on $X$.

\section{Discrete fuzzy topology}

Discrete fuzzy topology is the set that included all the fuzzy sets.

\section{Example 2:}

$$
\begin{aligned}
& X=\{x, y, z\}, \alpha=(0.8,0.9,0.7), \beta=(0.6,0.5,0.4), \\
& \delta=(0.3,0.3,0.2) \text { and } F=\{0, \alpha, \beta, \delta, 1\} .
\end{aligned}
$$

Now,

$$
\begin{aligned}
\alpha \wedge \beta=(0.8,0.9,0.7) \wedge(0.6,0.5,0.4)=(0.6,0.5,0.4)=\beta \in F \\
\alpha \wedge \delta=(0.8,0.9,0.7) \wedge(0.3,0.3,0.2)=(0.3,0.3,0.2)=\delta \in F \\
\beta \wedge \delta=(0.6,0.5,0.4) \wedge(0.3,0.3,0.2)=(0.3,0.3,0.2)=\delta \in F \\
\alpha \vee \beta=(0.8,0.9,0.7) \vee(0.6,0.5,0.4)=(0.8,0.9,0.7)=\alpha \in F \\
\text { And } \alpha \vee \delta=(0.8,0.9,0.7) \vee(0.3,0.3,0.2)=(0.8,0.9,0.7)=\alpha \in F \\
\beta \vee \delta=(0.6,0.5,0.4) \vee(0.3,0.3,0.2)=(0.6,0.5,0.4)=\beta \in F
\end{aligned}
$$

Also

$$
\begin{aligned}
\alpha \vee \beta \vee \delta & =(0.8,0.9,0.7) \vee(0.6,0.5,0.4) \vee(0.3,0.3,0.2) \\
& =(0.8,0.9,0.7) \\
& =\alpha \in F
\end{aligned}
$$

It is obvious $0,1 \in F$.

Therefore, $(X, F)$ is a fuzzy topological space.

\section{Example 3:}

Consider $X$ be an abstract set equipped with the family $F_{1}$ of fuzzy sets, where $F_{1}=\left\{v: v(x) \geq \frac{1}{2}, \forall x \in X\right\} \cup\left\{\alpha: \alpha\right.$ constant $\left.<\frac{1}{2}\right\}$

Then from the properties of fuzzy topological space

(i) $0,1 \in F_{1}$;

(ii) if $\rho_{1}, \rho_{2} \in F_{1}$; then $\rho_{1}(x) \wedge \rho_{1}(x) \Rightarrow\left(\rho_{1} \wedge \rho_{2}\right)(x) \geq \frac{1}{2}$ $\Rightarrow\left(\rho_{1} \wedge \rho_{2}\right) \in F_{1}$

i.e. finite intersection belongs to $F_{1}$.

(iii) $\rho_{i} \in F, \forall i \in j$, where $j$ denotes an index set.

so, $\underset{i \in j}{\vee} \rho_{i} \geq \frac{1}{2} \in F_{1}$

Hence, we conclude that the pair $(X, F)$ is a topological space.

\subsection{Closure Fuzzy Topological Space}

For any $\mu$ the closure of fuzzy topological space is denoted by $\bar{\mu}$ and is defined as the smallest closed fuzzy set that containing $\mu$.

Equivalent $\bar{\mu}$ is defined as following way: 


$$
\bar{\mu}=\{\alpha: \alpha \text { is F-closed and } \alpha \geq \mu\}
$$

Obviously, then $\bar{\mu}$ is always $F$-close.

Now we have discussed and proved some properties of Fuzzy closure operators.

\section{Properties of Closure Operator}

A map $\mu \rightarrow \bar{\mu}$ from $[0,1]^{X}$ into $[0,1]^{X}$ is said to be a closure operation if for all $\mu, \lambda \in[0,1]^{X}$ it satisfies the four list of properties:

(i) $\mu \leq \bar{\mu}$;

(ii) $\overline{\bar{\mu}} \leq \bar{\mu}$ (i.e. the closure operation is idempotent);

(iii) $\overline{\mu \vee \lambda}=\bar{\mu} \vee \bar{\lambda}$;

(iv) $\overline{0}=0$;

Proof (i):

From the definition of closure operator we know,

$$
\bar{\mu}=\wedge\{v: v \text { closed } \& v \geq \mu\} .
$$

Thus, $\mu \leq \bar{\mu}$.

Proof (ii):

Since $\overline{\bar{\mu}}$ is the smallest closed set containing $\bar{\mu}$ and $\bar{\mu}$ itself is closed, Then, $\overline{\bar{\mu}}=\bar{\mu}$.

\section{Proof (iii):}

Clearly $\bar{\alpha} \vee \bar{\beta}$ is closed.

Again,

$$
\begin{aligned}
& \bar{\alpha} \vee \bar{\beta} \geq \alpha \vee \beta \\
& \Rightarrow \overline{\bar{\alpha} \vee \bar{\beta}} \geq \overline{\alpha \vee \beta} \\
& \Rightarrow \bar{\alpha} \vee \bar{\beta} \geq \overline{\alpha \vee \beta}
\end{aligned}
$$

[Since $\bar{\alpha} \vee \bar{\beta}$ is closed, so $\overline{\bar{\alpha} \vee \bar{\beta}}=\bar{\alpha} \vee \bar{\beta}$ ]

Again,

$$
\begin{gathered}
\alpha \vee \beta \geq \alpha \\
\Rightarrow \overline{\alpha \vee \beta} \geq \bar{\alpha} \\
\text { Similarly, } \overline{\alpha \vee \beta} \geq \bar{\beta}
\end{gathered}
$$

From (a) and (b) we have,

$$
\overline{\alpha \vee \beta} \geq \bar{\alpha} \vee \bar{\beta}
$$

So from (1) and (2), we have,

$$
\bar{\alpha} \vee \bar{\beta}=\overline{\alpha \vee \beta} .
$$

Proof (iv):

Since the whole space $1 \in F$ is open, then its complement i.e. $1^{C}=0$, is closed

Also, $\overline{0}$ is closed.

So, we can write $\overline{0}=0$.

Definition. Fuzzy topology generated by closure operator is denoted by $F_{X}$ 
and is define by

$$
F_{X}=\left\{\mu \in[0,1]^{X}: \overline{1-\mu}=1-\mu\right\},
$$

Then $\left(X, F_{X}\right)$ is called closure of fuzzy topological space (f.t.s.) generated by closure operator.

\section{Theorem 4.1}

Suppose $\alpha, \beta$ are fuzzy sets in $X$ and $(X, F)$ is a fuzzy topological space. Then prove that $\bar{\alpha} \wedge \bar{\beta}=\overline{\alpha \wedge \beta}$.

Proof:

Clearly, $\bar{\alpha} \wedge \bar{\beta}$ is open. Again,

$$
\begin{aligned}
& \bar{\alpha} \wedge \bar{\beta} \leq \alpha \wedge \beta \\
& \Rightarrow \overline{\bar{\alpha} \wedge \bar{\beta}} \leq \overline{\alpha \wedge \beta} \\
& \Rightarrow \bar{\alpha} \wedge \bar{\beta} \leq \overline{\alpha \wedge \beta}
\end{aligned}
$$

Again,

$$
\begin{gathered}
\alpha \wedge \beta \leq \alpha \\
\Rightarrow \overline{\alpha \wedge \beta} \leq \bar{\alpha} \\
\text { Similarly, } \overline{\alpha \wedge \beta} \leq \bar{\beta}
\end{gathered}
$$

From (4) and (5) we have,

$$
\overline{\alpha \wedge \beta} \leq \bar{\alpha} \wedge \bar{\beta}
$$

From (3) and (6), we have,

$$
\bar{\alpha} \wedge \bar{\beta}=\overline{\alpha \wedge \beta} .
$$

\subsection{Interior of a Fuzzy Topological Space}

The smallest superior bound of all interior fuzzy sets of $\mu$ is called the interior of $\mu$, and is denoted by $\mu^{\circ}$.evidently, $\mu^{\circ} \in F^{X}$. So, $\mu^{\circ}$ is F-open.

Suppose $(X, F)$ be a fuzzy topological space and $I=[0,1]$, and $\mu, \lambda \in[0,1]^{X}$ where $\mu \geq \lambda$. Then $\lambda$ is defined as an interior fuzzy set of $\mu$ iff for $\rho \in F_{X}$ such that $\mu \geq \rho \geq \lambda$.

Theorem 4.2

Assume $(X, F)$ be a fuzzy topological space. Then

(i) $0^{\circ}=0,1^{\circ}=1$

(ii) $\mu^{\circ} \leq \mu$

(iii) $\mu^{\circ \circ}=\mu^{\circ}$

(iv) $(\mu \wedge v)^{\circ}=\mu^{\circ} \wedge v^{\circ}$

Proof (i):

Since, the interior of any set joining of all open subset contained in this set.

Now, the empty set 0 and the whole space 1 of an f.t.s. is open.

Thus $0^{\circ}=0,1^{\circ}=1$.

Proof (ii):

From the definition of interior of a set $\mu$, the combination of all open sub- 
sets included in $\mu$, denoted by $\mu^{\circ}$, i.e.

$$
\mu^{\circ}=\vee\{v \text { open } \& v \leq \mu\}
$$

Hence we conclude $\mu^{\circ} \leq \mu$.

Proof (iii):

Since $\mu^{\circ}$ itself is open and $\mu^{\circ \circ}$ is the greatest open set contained in $\mu^{\circ}$. So evidently, $\mu^{\circ \circ}=\mu^{\circ}$ (Proved).

Proof (iv):

Since $(\mu \wedge \lambda)^{\circ} \leq \mu^{\circ}$ and

$$
(\mu \wedge \lambda)^{\circ} \leq \lambda^{\circ}
$$

So,

$$
(\mu \wedge \lambda)^{\circ} \leq \mu^{\circ} \wedge \lambda^{\circ}
$$

On the other hand,

$\mu^{\circ} \wedge \lambda^{\circ} \leq \mu \wedge \lambda$, of which the open set $\mu^{\circ} \wedge \lambda^{\circ}$ hold in $\mu \wedge \lambda$;

Hence, $\mu^{\circ} \wedge \lambda^{\circ}$ must be contained in the largest open set $(\mu \wedge \lambda)^{\circ}$

$$
\text { i.e. } \mu^{\circ} \wedge \lambda^{\circ} \leq(\mu \wedge \lambda)^{\circ}
$$

From (7) and (8) we conclude that

$$
(\mu \wedge \lambda)^{\circ}=\mu^{\circ} \wedge \lambda^{\circ} \quad \text { (Proved) }
$$

\section{Theorem 4.3}

Assume $\alpha$ and $\beta$ be two fuzzy sets in an f.t.s.. Then $\alpha^{\circ} \vee \beta^{\circ}=(\alpha \vee \beta)^{\circ}$.

Proof:

$\alpha^{\circ}$ is open and $\beta^{\circ}$ is open .

So $\alpha^{\circ}=\alpha$, and $\beta^{\circ}=\beta$.

Therefore $\alpha^{\circ} \vee \beta^{\circ}=(\alpha \vee \beta)$

Then, $\left(\alpha^{\circ} \vee \beta^{\circ}\right)^{\circ}=(\alpha \vee \beta)^{\circ}$

Again, $\alpha^{\circ} \vee \beta^{\circ}$ is open, so $\left(\alpha^{\circ} \vee \beta^{\circ}\right)^{\circ}=\alpha^{\circ} \vee \beta^{\circ}$

Therefore, $\alpha^{\circ} \vee \beta^{\circ}=(\alpha \vee \beta)^{\circ}$

Hence, $\alpha^{\circ} \vee \beta^{\circ}=(\alpha \vee \beta)^{\circ}$ (Proved).

\subsection{Boundary of Fuzzy Topological Space}

For $\mu \in[0,1]^{X}$, the fuzzy boundary is denoted by $\mu^{b}$ is define to be the minimum of all F-closed sets $\rho$ with the property $\rho(x) \geq \bar{\mu}(x), \forall x \in X$ for which we have $[\bar{\mu} \wedge(1-\mu)](x)>0$.

Obviously, $\mu^{b}$ is F-closed and $\mu^{b} \leq \bar{\mu}$

\section{Fuzzy point}

For any fuzzy set, $\mu_{\beta} \in[0,1]^{X}$, fuzzy point is described as,

$$
\mu_{\beta}(x)= \begin{cases}\beta & \text { if } x=x_{\circ} \\ 0 & \text { otherwise }\end{cases}
$$

$x_{\circ}$ is the support of the fuzzy point $\mu_{\beta}$. 


\subsection{Neighborhood of Fuzzy Topological Space}

Suppose $(X, F)$ be a fuzzy topological space, if $\mu, \lambda \in[0,1]^{X}$ and $\mu \geq \lambda$ then $\mu$ is said to be a fuzzy neighborhood (in short n.b.d) of $\lambda$ if there is a $\gamma \in F_{X}$ so that $\mu \geq \gamma \geq \lambda$. Example:

We know if $(X, F)$ is a fuzzy topological space, and $\alpha, \beta \in F$, then $\beta$ is called fuzzy n.b.d. of $\alpha$ if and only if $\alpha \leq \beta$.

Now, let

$$
\begin{gathered}
X=\{u, v, w\}, F=\{0,1, \alpha, \beta, \gamma\}, \alpha=(0.5,0.3,0.2), \\
\beta=(0.7,0.1,0), \gamma=(0.5,0.1,0) . \\
F_{1 \alpha} \equiv(0.5,0.1,0.1) \leq \alpha \\
F_{2 \alpha} \equiv(0.0,0.3,0.0) \leq \alpha \\
F_{3 \alpha} \equiv(0.0,0.0,0.2) \leq \alpha
\end{gathered}
$$

where $F_{1 \alpha}, F_{2 \alpha}$ and $F_{3 \alpha}$ denote fuzzy points.

Here, $F_{1 \alpha} \vee F_{2 \alpha} \vee F_{3 \alpha}=\alpha$, but $F_{1 \alpha} \vee F_{2 \alpha} \vee F_{3 \alpha} \neq \beta$.

Also since,

$$
\begin{aligned}
& F_{1 \alpha} \leq \beta \\
& F_{2 \alpha} \leq \beta \\
& F_{3 \alpha} \leq \beta
\end{aligned}
$$

So, we conclude that $\alpha \leq \beta$.

Therefore $\beta$ is a fuzzy n.b.d. of $\alpha$.

\subsection{Continuous Function of a Fuzzy Topological Space}

Suppose $\left(X, F_{1}\right)$ and $\left(Y, F_{2}\right)$ be two fuzzy topological space and let $F:\left(X, F_{1}\right) \rightarrow\left(Y, F_{2}\right)$ be a function from $X$ into $F$. Then $F$ is said to be continuous at a point $x \in X$ if the inverse image $F^{-1}(v)$ of all $F_{2}$ open subset $v$ of $Y$ is a $F$ such that $F^{-1}(v) \in F_{1}$. Figure 6 displays the continuous open fuzzy set.

\section{Theorem 4.5}

Let $\left(X, F_{1}\right)$ and $\left(Y, F_{2}\right)$ be two fuzzy topological spaces. Then a function $F:\left(X, F_{1}\right) \rightarrow\left(Y, F_{2}\right)$

is continuous if

$$
F(\bar{\mu}) \leq \overline{F(\mu)} \text { for all } \mu \in I^{Y} .
$$

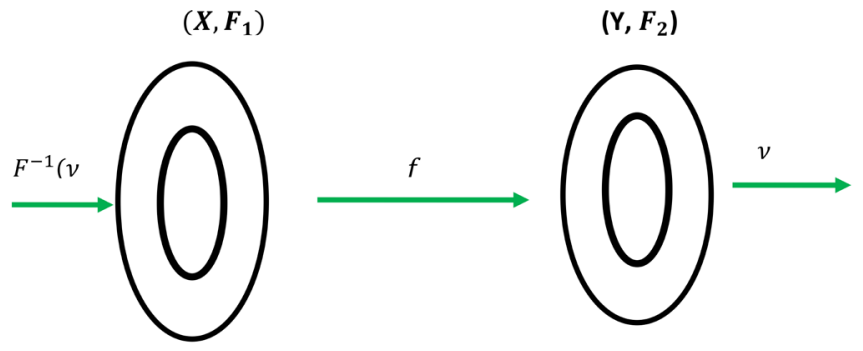

Figure 6. Continuous Open fuzzy set. 


\section{Proof:}

Let $\mu_{1} \in I^{Y}$ be such that $\mu_{1}^{C} \in F_{2}$ and put $\mu=F^{-1}\left(\mu_{1}\right)$, then

$F(\bar{\mu}) \leq \overline{F(\mu)}=\overline{F\left(F^{-1}\left(\mu_{1}\right)\right)} \leq \overline{\mu_{1}}=\mu_{1}$ So, But $\bar{\mu}$ is such that $\mu \leq \bar{\mu}$.

So, $\mu=\bar{\mu}$ and $\mu$ is closed,

I.e. $F^{-1}\left(\mu_{1}\right)$ is closed,

I.e. $F$ is continuous

Conversely, let $\mathrm{f}$ is fuzzy continuous. We must show that for any $\mu \in I^{X}$. $F(\bar{\mu}) \leq \overline{F(\mu)}$

We know that

$$
F(\bar{\mu})=\inf \left\{v \in I^{Y}: F^{-1}(v) \geq \bar{\mu}\right\}
$$

and $\overline{F(\mu)}=\inf \left\{\xi \in I^{Y}: \xi^{C} \in F_{2}, \xi \geq F(\mu)\right\}$

It suffices to prove that for all $\xi \in I^{Y}$ such that $\xi^{C} \in F_{2}$ and $\xi \geq F(\mu)$, there exists $v \in I^{Y}$ such that $F^{-1}(v) \geq \bar{\mu}$ and $\xi \geq v$.

Let, $\xi \in I^{Y}$ be such that $\xi \geq v$ and $\xi \geq F(\mu)$.

Put $v=\xi$, then $\xi \geq v$ and $f^{-1}(\xi) \geq F^{-1}(f(\mu)) \geq \mu$ and since $\xi^{C} \in F_{2}$ and $F$ is fuzzy continuous implies that $F^{-1}(\xi) \geq \bar{\mu}$.

Hence the theorem is proved.

\subsection{Homomorphism of Fuzzy Topological Space}

Let $F:\left(X, F_{1}\right) \rightarrow\left(Y, F_{2}\right)$ be a function. Then

i) $F$ is called continuous if $\forall v \in F_{2}, F^{-1}(v) \in F_{1}$.

ii) $F$ is called open if $\forall \mu \in F_{1}, F(\mu) \in F_{2}$.

iii) $F$ is called closed if $\forall F_{1}$-closed set $\mu, F(\mu)$ is $F_{2}$-closed.

iv) $F$ is called homomorphism if $F$ is bijective (i.e. one-one onto) and biconditional. It means that, both $f$ and $F^{-1}$ are continuous.

\section{Dense of fuzzy topological spaces}

Let $(X, F)$ be a fuzzy topological space. Some definitions on this space are given below:

\section{Definition 1.}

A fuzzy set $\mu$ is said to be fuzzy dense or everywhere dense if and only if $\bar{\mu}=1$.

\section{Definition 2.}

A fuzzy set $\mu$ is said to be fuzzy-nowhere dense if and only if $(\bar{\mu})^{C}=1$.

\section{Example:}

$\operatorname{int}(\bar{\mu})=0$, that is if the interior of closure of any fuzzy set $\mu$ is empty, then $\mu$ is called nowhere dense in $X$,

\section{Definition 3.}

Any fuzzy set $\mu$ is said to be fuzzy boundary (F-boundary) if and only if $\overline{1-\mu}=1$.

\section{Some Results Obtained from Definitions}

(i) If $\lambda \geq \mu$ and $\lambda$ is $F$-dense then $\mu$ is $F$-dense too. 


\section{Proof:}

Since, $\lambda \geq \mu$

$$
\begin{aligned}
& \Rightarrow \mu \leq \bar{\lambda} \\
& \Rightarrow \bar{\mu} \leq \overline{\bar{\lambda}} \\
& \Rightarrow \bar{\mu} \leq \bar{\lambda} \quad[\overline{\bar{\lambda}}=\bar{\lambda}]
\end{aligned}
$$

Since, $\bar{\lambda}=1$, thus $\bar{\mu}=1$.

Thus $\mu$ is $F$-dense too.

(ii) If $\lambda \leq \mu$ and $\mu$ is $F$-boundary, consequently then $\lambda$ is $F$-boundary too.

Proof:

Since $\lambda \leq \mu$

$$
\begin{aligned}
& \Rightarrow 1-\lambda \geq 1-\mu \\
& \Rightarrow \overline{1-\lambda} \geq \overline{1-\mu}
\end{aligned}
$$

So, $\overline{1-\mu}=1$, (Since $\mu$ is $F$-boundary).

Thus, $\overline{1-\lambda}=1$.

Thus, $\lambda$ is $F$-boundary too

(iii) If $\mu$ is $F$-nowhere dense and $\mu \geq \lambda$, then $\lambda$ is $F$-nowhere dense.

Proof:

Given, $\lambda \leq \mu$

$$
\begin{aligned}
& \lambda \leq \bar{\mu} \\
& \Rightarrow 1-\lambda \geq 1-\bar{\mu} \\
& \Rightarrow \overline{1-\lambda} \geq \overline{1-\bar{\mu}}
\end{aligned}
$$

Since $\mu$ is $F$-nowhere dense, which implies $\overline{1-\lambda}=1$, from which we conclude that $\lambda$ is $F$-nowhere dense.

(iv) If $\mu$ is $F$-nowhere dense, then so is $\bar{\mu}$.

Proof:

Since $\mu \leq \bar{\mu}$

$$
\begin{aligned}
& \Rightarrow 1-\mu \geq 1-\bar{\mu} \\
& \Rightarrow \overline{1-\mu} \geq \overline{1-\bar{\mu}} \\
& \Rightarrow \overline{\mu^{C}} \geq \overline{(\bar{\mu})^{C}}
\end{aligned}
$$

Since $\mu$ is nowhere dense, so $\overline{\mu^{C}}=1$.

Hence from (9) we conclude that $\bar{\mu}$ is $F$-nowhere dense.

(v) If $\mu \in[0,1]^{X}$ is F-dense and $\lambda \in F$, then $\lambda \leq \overline{\mu \wedge \lambda}$.

Proof:

We know from the properties of closure operation

$$
\mu \wedge \lambda \leq \overline{\mu \wedge \lambda}
$$

Also, $\overline{\mu \wedge \lambda} \leq \bar{\mu} \wedge \bar{\lambda}$

Thus,

$$
\mu \wedge \lambda \leq \overline{\mu \wedge \lambda} \leq \bar{\mu} \wedge \bar{\lambda}
$$


Also,

$$
\bar{\mu} \wedge \bar{\lambda} \leq \bar{\lambda}
$$

And we know,

$$
\bar{\lambda} \geq \lambda
$$

Hence, from (10), (11), and (12) we conclude that

$$
\overline{\mu \wedge \lambda} \geq \lambda \text {. }
$$

\section{Proposition 4.1}

If $\mu$ is $F$-dense then, $(1-\mu)^{\circ}=0$. That is

Proof:

We claim from the definition of interior fuzzy set,

$$
(1-\mu)^{\circ}=1-\bar{\mu} \text {. }
$$

Now,

$$
\begin{aligned}
1-\bar{\mu} & =1-\wedge\{\tau:(1-p) \in F, p \geq \mu\} \\
& =\vee\{(1-p) \in F: v \geq \tau\} \\
& =\vee\{\tau \in F: 1-\tau \geq \mu\} \\
& =\vee\{\tau \in F: 1-\mu \geq \tau\} \\
& =(1-\mu)^{\circ}
\end{aligned}
$$

But since $\mu$ is $F$-dense, so, $\bar{\mu} \equiv 1$.

Hence, $1-\bar{\mu}=0$.

\subsection{Base and Subbase of the Fuzzy Topological Spaces}

\section{Base}

Let $(X, F)$ be a fuzzy topological space and $P$ is a sub family of $F$, then $P$ is called a base of $F$ if and only if every member of $F$ can be represent as supremum of member of $P$.

Example:

Let $X=\{x, y\}$, and $\alpha, \beta, \gamma \in I^{X}$, where $\alpha=(0.3,0.6), \quad \beta=(0.7,0.5)$, $\gamma=(0.7,0.6)$ and

$(X, F)$ be a fuzzy topological space, where $F=\{0,1, \alpha, \beta, \gamma, \delta\}$ and $B=\{0, \alpha, \beta, \lambda, 1\}$.

Then, $0, \alpha, \beta, \gamma$ and 1 are in $P$.

Now, $\gamma=(0.7,0.6)=\alpha \vee \beta$

Hence $P$ is a base for $F$.

\section{Subbase}

Suppose $(X, F)$ is a fuzzy topological space and $B$ is a non empty subset of $F$, then $B$ is called a subase of $F$, if and only if the finite intersection of members of $B$ forms a base for $F$.

Example:

Assume $X=\{x, y\}$ and $\alpha, \beta, \gamma, \delta \in I^{X}$ where, $\alpha=(0.2,0.6)$, 
$\beta=(0.3,0.5), \quad \gamma=(0.2,0.5)$ and $\delta=(0.3,0.6)$ and $\delta=(0.3,0.6)$.

Then $(X, F)$ is a fuzzy topological space, where $F=\{0,1, \alpha, \beta, \gamma, \delta\}$ and $B=\{\alpha, \beta\}$ is a subbase and $P=\{0,1, \alpha, \beta, \gamma\}$ is a base of $F$.

\subsection{Hausdroff Fuzzy Topological Spaces}

An fuzzy topological space $(X, F)$ is said to be Hausdroff iff $\forall x, y \in X$, $x \neq y$, there exist $\mu, \lambda \in F$ so that $\mu(x)=\lambda(y)=1$ and $\mu \wedge \lambda=0$.

\section{Theorem 4.7}

Let $(X, F)$ is a Hausdroff fuzzy topological space, while $\left(Y, F_{1}\right)$ a fuzzy topological space and $F: X \rightarrow Y$ is an $F$ open bijection, then $\left(Y, F_{1}\right)$ is also a Hausdroff fuzzy topological space.

\section{Proof:}

Let $y_{1}, y_{2} \in Y$ and $y_{1} \neq y_{2}$.

Let, $y_{1}=f\left(x_{1}\right)$ and $y_{2}=f\left(x_{2}\right)$. Since by hypothesis $(X, F)$ is a Hausdroff topological space, then there exist $\mu, \lambda \in F$ such that $\mu\left(x_{1}\right)=\lambda\left(x_{2}\right)=1$ and $\mu \wedge \lambda=0$.

Now, $f(\mu)$ and $f(\lambda)$ both belong to $F_{1}$, since $f$ is an $F$-open map.

Also $f(\mu \wedge \lambda)=f(\mu) \wedge f(\lambda)=F(0)=0$.

Finally,

$$
f(\mu)\left(y_{2}\right)=\sup _{x \in f^{-1}\left(y_{1}\right)} \mu(x)=1 \text { and } f(\lambda)\left(y_{1}\right)=\sup _{x \in f^{-1}\left(y_{2}\right)} \lambda(x)=1
$$

Which satisfy all the condition of Hausdroff fuzzy topological space.

Hence, indeed $\left(Y, F_{1}\right)$ is a Hausdroff fuzzy topological space.

\section{Conclusion}

Our main aim of this study is to make simple for the reader to understand the relation between the fuzzy set and topological spaces. For that purpose we have attempted to provide more fundamental definition of these two topics. In the fuzzy topological spaces section we have represented definition and provide proved of some theorem. The presented results in this analysis signify that large numbers of the fundamental ideas from general topology may be expanded enthusiastically to topological fuzzy spaces. Even though the fuzzy sets hypothesis belongs in a developing phase, now a days it is undertaken of having spacious precious applications.

\section{Conflicts of Interest}

The authors declare no conflicts of interest regarding the publication of this paper.

\section{References}

[1] Black, M. (1937) Vagueness: An Exercise in Logical Analysis. Philosophy of Science, 4, 427-455. (Reprinted in International Journal of General Systems, 17(2-3), 107128, 1990) https://doi.org/10.1086/286476 
[2] Zadeh, L.A. (1965) Fuzzy Sets. Information and Control, 8, 338-353. https://doi.org/10.1016/S0019-9958(65)90241-X

[3] Chang, C.L. (1968) Fuzzy Topological Spaces. Journal of Mathematical Analysis and Applications, 24, 182-190. https://doi.org/10.1016/0022-247X(68)90057-7

[4] Michálek, J. (1975) Fuzzy Topologies. Kybernetika, 11, 345-354.

[5] Lowen, R. (1976) Fuzzy Topological Spaces and Fuzzy Compactness. Journal of Mathematical Analysis and Application, 56, 621-633. https://doi.org/10.1016/0022-247X(76)90029-9

[6] Hutton, B. (1980) Products of Fuzzy Topological Spaces. Topology and Its Applications, 11, 59-67. https://doi.org/10.1016/0166-8641(80)90017-6

[7] Chang, C.L. (1985) Fuzzy Topological Spaces. Journal of Mathematical Analysis and Applications, 110, 141-178. https://doi.org/10.1016/0022-247X(85)90340-3

[8] Zimmermann, H.J. (1985) Fuzzy Set Theory and Its Applications. Kluwer, Boston, 11-17. https://doi.org/10.1007/978-94-015-7153-1 2

[9] Papageorgiou, N.S. (1985) Fuzzy Topology and Fuzzy Multifunctions. Journal of Mathematical Analysis and Applications, 109, 397-425. https://doi.org/10.1016/0022-247X(85)90159-3

[10] Klir, G.J. and Yuan, B. (1995) Fuzzy Sets and Fuzzy Logic: Theory and Applications. Prentice Hall, Hoboken, 1-49.

[11] Lee, S.J. and Lee, E.P. (2000) The Category of Intuitionistic Fuzzy Topological Spaces. Bulletin of the Korean Mathematical Society, 37, 63-76.

[12] Yan, C. and Wang, X. (2010) Intuitionistic I-Fuzzy Topological Spaces. Czechoslovak Mathematical Journal, 60, 233-252. https://doi.org/10.1007/s10587-010-0013-7

[13] Shi, W.Z. and Liu, K. (2007) A Fuzzy Topology for Computing the Interior, Boundary, and Exterior of Spatial Objects Quantitatively in GIS. Computers \& Geosciences, 33, 898-915. https://doi.org/10.1016/j.cageo.2006.10.013

[14] Kandil, A., Saleh, S. and Yakout, M.M. (2012) Fuzzy Topology on Fuzzy Sets: Regularity and Separation Axioms. American Academic \& Scholarly Research Journal, 4, $1-12$.

[15] Akray, I. (2013) On Fuzzy Topological Spaces Induced by a Given Function. Asian Journal of Fuzzy and Applied Mathematics, 1, 44-50.

[16] Aygunoglu, A., Cetkin, V. and Aygun, H. (2014) An Introduction to Fuzzy Soft Topological Spaces. Hacettepe Journal of Mathematics and Statistics, 43, 197-208.

[17] Chamua, H.C. and Chetia, B.C. (2015) Application of Fuzzy Topological Relation in Flood Prediction. International Journal of Computer Applications, 122, 1-3. https://doi.org/10.5120/21710-4830

[18] Berge, C. (2010) Topological Spaces. Oliver and Boyd, Edinburg and London, 54-56.

[19] Simmons, G.F. (1963) Introduction to Topology and Modern Analysis. McGrawHill Book Company, Inc., New York, 92-99.

[20] Lipschotz, S. (2011) General Topology, Schaum's Outlines. McGraw-Hill Education, New York, 66-90. 